\title{
Article
}

\section{Low-Energy Coulomb Excitation for the Shell Model}

\author{
Marco Rocchini ${ }^{1}{ }^{(0 *}$ and Magda Zielińska ${ }^{2}$ (1)* \\ 1 Department of Physics, University of Guelph, N1G 2W1 Guelph, Canada \\ 2 IRFU, CEA, Université Paris-Saclay, F-91191 Gif-sur-Yvette, France \\ * Correspondence: mrocchin@uoguelph.ca (M.R), magda.zielinska@cea.fr (M.Z.)
}

\begin{abstract}
Low-energy Coulomb excitation is capable of providing unique information on static electromagnetic moments of short-lived excited nuclear states, including non-yrast states. The process selectively populates low-lying collective states and is therefore ideally suited to study phenomena such as shape coexistence and development of exotic deformation (triaxial or octupole shapes). Historically, these experiments were restricted to stable isotopes. However, the advent of new facilities providing intense beams of short-lived radioactive species has opened the possibility to apply this powerful technique to a much wider range of nuclei. We discuss the observables that can be measured in a Coulomb-excitation experiment, their relation to nuclear structure parameters with an emphasis on the nuclear shape, and present some recent examples of Coulomb-excitation studies that provided outcomes relevant for the Shell Model.
\end{abstract}

Keywords: Nuclear Structure; Low-Energy Coulomb Excitation; Shell Model

\section{Introduction}

Among the multitude of experimental techniques used in nuclear-structure physics, low-energy Coulomb excitation is one of the oldest and, still to this day, one of the most widely employed. The reason for its success is twofold. On the one hand, this technique requires ion beams with relatively low energy (a few $\mathrm{MeV} / \mathrm{A}$ ) and the large cross sections of the Coulomb-excitation process can compensate for low beam intensity. For these reasons, it was widely used for experimental nuclear-structure studies in their early days and, at present, leads the way at new-generation ISOL radioactive ion beam (RIB) facilities. On the other hand, low-energy Coulomb excitation is particularly sensitive to nuclear collective properties, such as the shape. Specifically, this method can be used to determine reduced transition probabilities between low-lying states, and their spectroscopic quadrupole moments. As it relies on the well-known electromagnetic interaction, all these observables can be extracted in a model-independent way. Furthermore, the unique and model-independent information on relative signs of $E 2$ matrix elements, achievable solely with this technique, makes it possible to link transitional and diagonal $E 2$ matrix elements to Hill-Wheeler parameters $\left(\beta_{2}, \gamma\right)$ describing a quadrupole shape, via non-energy weighted quadrupole sum rules [1]. Hence, low-energy Coulomb excitation constitutes a powerful tool to study phenomena such as shape coexistence, shape transitions, superdeformation, and octupole collectivity (see Refs. [2-4] for recent examples).

This paper aims to outline how the results of low-energy Coulomb-excitation measurements can be used to benchmark the Shell Model and inspire further theoretical developments. In the next section, we briefly introduce the method and discuss firstand higher-order effects giving rise to sensitivity to transitional and diagonal electromagnetic matrix elements. The following section presents examples of low-energy Coulombexcitation experiments that provided outcomes particularly relevant for the Shell Model. We do not aim to provide a comprehensive review of low-energy Coulomb-excitation studies, which can be found elsewhere (see for instance Refs. [5,6]). 


\section{Excitation}

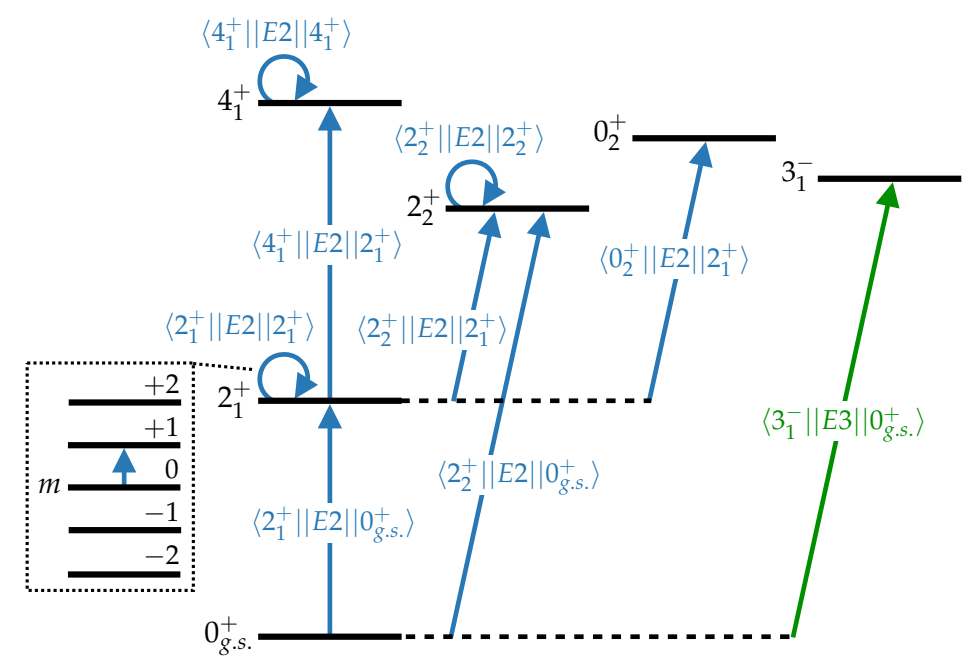

\section{De-excitation}

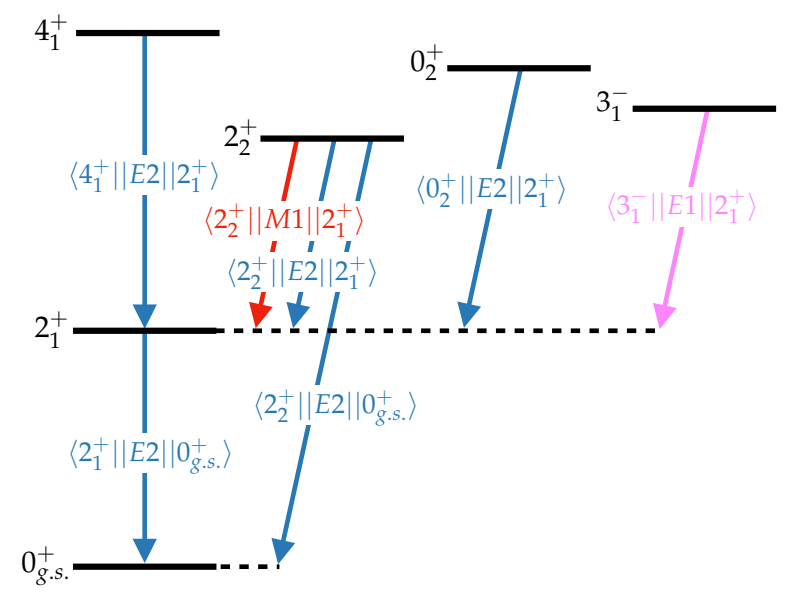

Figure 1. Low-lying level scheme of a fictitious even-even nucleus outlining dominant excitation (left) and de-excitation (right) patterns in low-energy Coulomb excitation. The transitions are labelled with the corresponding matrix elements. The inset on the left depicts the magnetic substates $m$ of the $2_{1}^{+}$state and illustrates the reorientation effect. Some allowed transitions are neglected for simplicity. (Color online.)

\section{Basics of Low-Energy Coulomb Excitation}

Coulomb excitation is an inelastic scattering process, in which the two colliding nuclei are excited via the mutually-generated, time-dependent electromagnetic field. If the distance between the collision partners is sufficiently large, the short-range nuclear interaction has a negligible influence on the excitation process, which is governed solely by the well-known electromagnetic interaction. This condition can be quantified using the Cline's safe distance criterion [7], appropriate for heavy nuclei, which states that if the distance of closest approach between the surfaces of the collision partners exceeds $5 \mathrm{fm}$, contributions from the nuclear interaction to the observed excitation cross sections are below $0.5 \%$. The excitation cross sections depend on electromagnetic matrix elements coupling the low-lying states in the nucleus of interest, including diagonal E2 matrix elements related to spectroscopic quadrupole moments. The decay of Coulomb-excited states is governed by the same set of electromagnetic matrix elements, although the influence of specific matrix elements on the excitation and decay processes may be very different, as illustrated by Figure 1. Namely, low-energy Coulomb excitation favours the population of collective states through E2 and E3 transitions, while other multipolarities have usually a small impact on the measured cross sections (see Ref. [8] for further details). The M1 and E1 multipolarities, however, remain important in the de-excitation process. The quantities measured in low-energy Coulomb-excitation experiments are, most commonly, $\gamma$-ray yields in coincidence with at least one of the collision partners. It is, however, also possible to measure Coulomb-excitation cross sections by detecting only scattered particles or only $\gamma$ rays.

While Coulomb-excitation cross sections can be calculated using a full quantummechanical treatment, a semi-classical approach is typically employed to overcome difficulties arising from the long-range of the Coulomb interaction and complex level schemes of the colliding nuclei. In this approach, introduced by K. Alder and A. Winther [9], the relative motion of collision partners is described using classical equations, and the quantal treatment is limited to the excitation process. The validity of this procedure, which provides a significant simplification of the calculations without a relevant loss of accuracy, stems from the fact that the interaction in the Coulomb-excitation process is dominated by the Rutherford term. For the semi-classical approximation to be valid, the de Broglie wavelength associated with the projectile must be small compared to the distance 
of closest approach, and the energy transferred in the excitation process must be small compared with the total kinetic energy in the centre-of-mass reference system. These two conditions are well satisfied in low-energy Coulomb-excitation experiments involving heavy ions, but when light nuclei are involved (i.e. protons, deuterons, $\alpha$ particles), a full quantum-mechanical analysis is required.

\subsection{First-Order Effects}

If the interaction between the colliding nuclei is weak, i.e. the excitation probability is $\ll 1$, Coulomb-excitation amplitudes can be calculated within the first-order perturbation theory. In the first order, the cross section for the excitation of the state $I_{f}$ from the ground state $I_{\text {g.s. }}$ is proportional to the square of the transitional matrix element $\left\langle I_{f} \| E L|| I_{\text {g.s. }}\right\rangle$. Therefore, from the measured $I_{\text {g.s. }} \rightarrow I_{f}$ Coulomb-excitation cross section it is possible to extract the reduced transition probability $B\left(E L ; I_{g . s .} \rightarrow I_{f}\right)$.

The excitation process strongly depends on the kinematics and the mass and atomic numbers of the target and projectile nuclei. The first-order approximation is usually sufficiently accurate to describe the population of excited states from the ground state in experiments employing a light beam or a light target, or when small centre-of-mass scattering angles are used; examples of such recent studies are presented in Section 3.4. Larger kinetic energy, larger atomic numbers of the collision partners, and lower excitation energies enhance the excitation probability, leading to the appearance of higher-order effects in the excitation process.

\subsection{Higher-Order Effects}

If the electromagnetic field acting between the collision partners is strong enough and the collision process lasts a sufficiently long time, multi-step excitation becomes a possibility and higher-order contributions have to be taken into account. These contributions give rise to the experimental sensitivity to relative signs of transitional matrix elements and spectroscopic quadrupole moments of excited states, as described in the following.

\subsubsection{Multi-Step Excitation and Relative Signs}

To understand the importance of multi-step excitation it is useful to consider the population of two excited states $I^{\pi}=0_{2}^{+}, 4_{1}^{+}$in an even-even nucleus (see Figure 1). Because Coulomb excitation via an $E 0$ transition is strictly forbidden, two-step excitation is the only way to populate the $0_{2}^{+}$state. The $4_{1}^{+}$state can be Coulomb-excited in two ways: directly from the ground state, via an $E 4$ excitation, or with an $E 2$ two-step excitation through the first excited state. Since the probability of Coulomb-exciting a given state through an E4 transition is much smaller than through the E2 excitation [8], the twostep excitation is typically dominant ${ }^{1}$. Consequently, by measuring the intensities of the $4_{1}^{+} \rightarrow 2_{1}^{+}, 0_{2}^{+} \rightarrow 2_{1}^{+} \gamma$-ray transitions with respect to the $2_{1}^{+} \rightarrow 0_{1}^{+}$decay, and relating them to excitation cross sections, it is possible to extract the $B\left(E 2 ; 4_{1}^{+} \rightarrow 2_{1}^{+}\right)$and $B\left(E 2 ; 0_{2}^{+} \rightarrow 2_{1}^{+}\right)$ values.

In some cases, single- and multi-step excitations are comparable in magnitude; an example is the $2_{2}^{+}$state in an even-even nucleus (see Figure 1). This state can be populated by a direct $E 2$ transition from the ground state and by a two-step excitation through the first excited state. The total excitation probability for the $2_{2}^{+}$state can be written as:

$$
P\left(0_{g . s .}^{+} \rightarrow 2_{2}^{+}\right)=\left|a^{(1)}\left(0_{g . s .}^{+} \rightarrow 2_{2}^{+}\right)+a^{(2)}\left(0_{g . s .}^{+} \rightarrow 2_{1}^{+} \rightarrow 2_{2}^{+}\right)\right|^{2},
$$

1 In the extreme case of ${ }^{150} \mathrm{Nd}$, characterised by an enhanced $E 4$ strength $\left(\left\langle 4_{1}^{+}|| E 4|| 0_{\text {g.s. }}^{+}\right\rangle=0.22(12) e \mathrm{~b}^{2}[10]\right)$, the contribution from $E 4$ excitation is expected to reach $\approx 30 \%$ of the total cross section to populate the $4_{1}^{+}$state in backscattering of ${ }^{150} \mathrm{Nd}$ on a ${ }^{208} \mathrm{~Pb}$ target. Typical contributions are much lower. 
where $a^{(1)}, a^{(2)}$ are first- and second-order excitation amplitudes. Consequently, $P\left(0_{\text {g.s. }}^{+} \rightarrow\right.$ $\left.2_{2}^{+}\right)$includes a term related to one-step excitation $\left(\left\langle 2_{2}^{+}|| E 2|| 0_{\text {g.s. }}^{+}\right\rangle^{2}\right)$, one related to two-step excitation $\left(\left\langle 2_{2}^{+}|| E 2|| 2_{1}^{+}\right\rangle^{2}\left\langle 2_{1}^{+}|| E 2|| 0_{\text {g.s. }}^{+}\right\rangle^{2}\right)$ and the interference term

$$
\left\langle 2_{2}^{+}\|E 2\| 0_{\text {g.s. }}^{+}\right\rangle\left\langle 2_{2}^{+} \| E 2|| 2_{1}^{+}\right\rangle\left\langle 2_{1}^{+}\|E 2\| 0_{\text {g.s. }}^{+}\right\rangle .
$$

In this last term, at variance with all the others, the matrix elements are not squared. As the total Coulomb-excitation cross section will be different for a negative (destructive) and a positive (constructive) interference term, its sign becomes an observable.

More complex interference terms can influence the Coulomb-excitation cross sections if states are populated through several excitation patterns involving multiple intermediate states. As such terms include non-squared matrix elements, their appearance leads to the experimental sensitivity to relative signs of transitional matrix elements. A sign convention should be adopted to ensure consistent analysis and facilitate a comparison with model predictions. Usually, signs of all in-band transitional E2 matrix elements are assumed to be positive, and, for each band head, a positive sign is imposed for one of the transitions linking it with a state in the ground-state band. The signs of all remaining matrix elements can be determined relative to those.

The probability of exciting a state via a process involving two or more steps can be comparable to that of one-step excitation, depending, for instance, on the magnitude of the involved matrix elements. Also, multi-step excitation is enhanced for larger scattering angles and masses of the collision partners. Experiments aiming at extracting reduced transition probabilities between the ground state and an excited state are typically performed in conditions reducing multi-step excitations, by limiting the scattering angle in the forward direction and selecting a light collision partner. In contrast, if the relative signs of transitional matrix elements and reduced transition probabilities between excited states are the objective of the experiment, the detection of scattering particles at backward angles and the use of a heavy collision partner is preferable.

\subsubsection{Reorientation Effect and Spectroscopic Quadrupole Moments}

The reorientation effect [11] is another second-order effect in Coulomb excitation, which provides experimental sensitivity to spectroscopic quadrupole moments $\left(Q_{s}\right)$ of excited nuclear states. This effect essentially consists in a double-step excitation, in which the intermediate state is identical to the final state, but the magnetic substate is different (see Figure 1). For a given state $I^{\pi}$, reorientation produces a second-order correction to its Coulomb-excitation cross section, which is proportional to the diagonal matrix element $\left\langle I^{\pi}|| E 2|| I^{\pi}\right\rangle$, i.e. to $Q_{s}\left(I^{\pi}\right)$. Since this matrix element, and not its square, appears in the expression for cross section, also its sign is an observable. In favourable conditions, the reorientation effect may have a considerable influence on the measured $\gamma$-ray intensities. For example, in a recent study of ${ }^{74} \mathrm{Kr}$ Coulomb-excited on ${ }^{208} \mathrm{~Pb}$ [12], a change of sign of the $Q_{s}\left(2_{1}^{+}\right)$from negative to positive would result in a 1.8-fold increase of the $4_{1}^{+} \rightarrow$ $2_{1}^{+} / 2_{1}^{+} \rightarrow 0_{1}^{+}$intensity ratio measured in coincidence with $\mathrm{Kr}$ nuclei scattered at $130^{\circ}$ in the centre-of-mass frame.

The influence of the reorientation effect on Coulomb-excitation cross sections is often comparable to that of multi-step excitations. Consequently, the impact of the spectroscopic quadrupole moment can compete with, for instance, that of the sign of an interference term. This is why in early low-energy Coulomb-excitation measurements two values of the spectroscopic quadrupole moment were often reported: one corresponding to a positive sign of the $\left\langle 0_{1}^{+} \| E 2|| 2_{1}^{+}\right\rangle\left\langle 2_{1}^{+} \| E 2|| 2_{2}^{+}\right\rangle\left\langle 2_{2}^{+}\|E 2\| 0_{1}^{+}\right\rangle$interference term, and the other one for a negative sign. This ambiguity can be solved by measuring $\gamma$-ray yields as a function of the scattering angle, thus exploiting the different angular dependence of the two effects $[5,13]$. This approach, typically referred to as a differential Coulomb-excitation measurement, is often employed nowadays. The use of different beam-target combinations in the same experiment can also help to disentangle competing contributions to the cross sections, 
and more constraints can be provided by including known spectroscopic data (lifetimes, branching ratios, E2/M1 branching ratios) in the Coulomb-excitation data analysis.

\subsection{Quadrupole Sum Rules}

The nuclear shape can be inferred indirectly from transition probabilities or spectroscopic quadrupole moments, but this approach is not always unambiguous and generally depends on comparisons with models. An alternative model-independent approach, proposed by K. Kumar and D. Cline [1,7], exploits the specific properties of the electromagnetic multipole operators. As these operators are spherical tensors, their zero-coupled products are rotationally invariant. The expectation values of these products are observables, and they are strictly related to the parameters describing the shape of the charge distribution.

The electric quadrupole operator in the principal axis system can be represented using the variables $Q$ and $\delta$, whose expectation values are equivalent to the Hill-Wheeler parameters $\left(\beta_{2}, \gamma\right)$ describing the quadrupole shape. The simplest invariants read:

$$
\begin{aligned}
& \{E 2 \times E 2\}^{0}=\frac{1}{\sqrt{5}} Q^{2}, \\
& \left\{[E 2 \times E 2]^{2} \times E 2\right\}^{0}=-\sqrt{\frac{2}{35}} Q^{3} \cos 3 \delta .
\end{aligned}
$$

The expectation values of these invariants for a state $I_{n}$ can be expressed through $E 2$ matrix elements defined in the laboratory system. For instance:

$$
\begin{aligned}
& \left\langle I_{n}\left|Q^{2}\right| I_{n}\right\rangle=\frac{\sqrt{5}(-1)^{2 I_{n}}}{\sqrt{2 I_{n}+1}} \sum_{m} M_{n m} M_{m n}\left\{\begin{array}{ccc}
2 & 2 & 0 \\
I_{n} & I_{n} & I_{m}
\end{array}\right\}, \\
& \left\langle I_{n}\left|Q^{3} \cos 3 \delta\right| I_{n}\right\rangle=-\sqrt{\frac{35}{2}} \frac{(-1)^{2 I_{n}}}{2 I_{n}+1} \sum_{m l} M_{n l} M_{l m} M_{m n}\left\{\begin{array}{ccc}
2 & 2 & 2 \\
I_{n} & I_{m} & I_{l}
\end{array}\right\},
\end{aligned}
$$

where $M_{a b} \equiv\left\langle I_{a}|| E 2|| I_{b}\right\rangle$ and the expression in curly brackets is a $6 j$ coefficient. Higherorder invariants can be defined, such as $\left\langle Q^{4}\right\rangle$, which can be linked to the dispersion in $\left\langle Q^{2}\right\rangle$ via

$$
\sigma\left(Q^{2}\right)=\sqrt{\left\langle Q^{4}\right\rangle-\left(\left\langle Q^{2}\right\rangle\right)^{2}} .
$$

A similar definition applies to $\sigma\left(Q^{3} \cos 3 \delta\right)$. In principle, this approach can be extended to more complex, non-quadrupole shapes.

The invariants obtained from quadrupole sum rules provide a model-independent description of the nuclear shape in the intrinsic reference system. However, the experimental determination of such invariants requires numerous matrix elements to be known. While for the lowest-order shape invariant, $\left\langle Q^{2}\right\rangle$, all matrix elements enter the sum in squares, this is not true for most higher-order invariants. In particular, the $\left\langle Q^{3} \cos 3 \delta\right\rangle$ invariant is constructed from triple products of $E 2$ matrix elements, $\left\langle I_{n}|| E 2|| I_{l}\right\rangle\left\langle I_{l}|| E 2|| I_{m}\right\rangle\left\langle I_{m}|| E 2|| I_{n}\right\rangle$, where $\left|I_{n}\right\rangle$ is the state in question and $\left|I_{l}\right\rangle$ and $\left|I_{m}\right\rangle$ are the intermediate states. The diagonal matrix elements (i.e. $\left|I_{l}\right\rangle=\left|I_{m}\right\rangle$ ) and their signs are necessary to extract this invariant, as well as the relative signs of all relevant transitional matrix elements.

While the sums in Eqs. 5, 6 formally run over all intermediate states that can be reached from the state in question via a single $E 2$ transition, usually only a few key states contribute to the invariant. In particular, for the ground state of an even-even nucleus, the contributions to $\left\langle Q^{2}\right\rangle$ are dominated by the coupling to the $2_{1}^{+}$state, which typically amounts to well over $90 \%$ of the total. Similarly, the largest contributions to $\left\langle Q^{3} \cos 3 \delta\right\rangle$ for the ground state come from the $\left\langle 0_{1}^{+} \| E 2|| 2_{1}^{+}\right\rangle\left\langle 2_{1}^{+}|| E 2|| 2_{1}^{+}\right\rangle\left\langle 2_{1}^{+}\|E 2\| 0_{1}^{+}\right\rangle$and $\left\langle 0_{1}^{+}|| E 2|| 2_{1}^{+}\right\rangle\left\langle 2_{1}^{+}|| E 2|| 2_{2}^{+}\right\rangle\left\langle 2_{2}^{+}|| E 2|| 0_{1}^{+}\right\rangle$products. The situation becomes much more complicated for excited states, and the number of intermediate states that need to be included 
in the sum rules varies from one case to another. While theoretical approaches can, in principle, provide a complete set of electromagnetic matrix elements, this is not always true for experiments. Systematic studies employing the Shell Model addressed this convergence issue [14-16]. The contributions of individual products of matrix elements to the experimentally determined invariants have also been analysed in some cases [15,17-19].

\section{Examples of Recent Low-Energy Coulomb-Excitation Studies Relevant for the Shell Model}

The examples of experimental studies presented in this section illustrate the variety of nuclear-structure questions, relevant for the Shell Model, that can be addressed using low-energy Coulomb excitation. We focus on the region of mid-mass nuclei between Ca and Sm, and direct the reader to Ref. [6] for a discussion of other noteworthy examples, particularly concerning lighter nuclei important for astrophysical processes.

\subsection{Superdeformation in ${ }^{42} \mathrm{Ca}$}

The potential of Coulomb excitation as a tool to study superdeformation has been demonstrated in the very first experiment using the AGATA $\gamma$-ray tracking array [20]. The superdeformed (SD) structure in ${ }^{42} \mathrm{Ca}$ was populated following Coulomb excitation of a ${ }^{42} \mathrm{Ca}$ beam on ${ }^{208} \mathrm{~Pb}$ and ${ }^{197} \mathrm{Au}$ targets $[3,19]$. From the measured $\gamma$-ray intensities, magnitudes and relative signs of numerous $E 2$ matrix elements coupling the low-lying states in ${ }^{42} \mathrm{Ca}$ were determined. In particular, two key pieces of information were obtained for the first time, which confirm that the band built on the $0_{2}^{+}$state in ${ }^{42} \mathrm{Ca}$ has a SD character at low spin: the spectroscopic quadrupole moment of the $2_{2}^{+}$state, which corresponds to $\beta_{2}=0.48(16)$, as well as the enhanced $B\left(E 2 ; 2_{2}^{+} \rightarrow 0_{2}^{+}\right)=15_{-4}^{+6}$ W.u. value. As discussed in Ref. [13], even though the $2_{2}^{+} \rightarrow 0_{2}^{+}$transition is too weak to be observed and prior to the study of Refs. $[3,19]$ only an upper limit for the branching ratio was known, the corresponding matrix element has a strong influence on excitation cross sections of the observed states, and hence, it could be determined from the intensities of other transitions measured in the Coulomb-excitation experiment.

The obtained transitional and diagonal E2 matrix elements were further interpreted in terms of quadrupole invariants of the $0_{1,2}^{+}$and $2_{1,2}^{+}$states, leading to the conclusion that the spherical ground state of ${ }^{42} \mathrm{Ca}$ exhibits large fluctuations into the $\beta_{2}-\gamma$ plane, while the excited structure has a large quadrupole deformation of $\beta_{2}=0.43(4)$ for the $\mathrm{O}_{2}^{+}$state, comparable to those deduced from lifetime measurements for other SD bands in this mass region. The important increase of the $\left\langle Q^{2}\right\rangle$ quadrupole invariant for the $2_{1}^{+}$ state with respect to that for the ground state was attributed to the mixing of the $2^{+}$states. Additionally, the triaxiality parameter $\langle\cos 3 \delta\rangle$ obtained for the $0_{2}^{+}$state, corresponding to $\gamma=\left(13_{-6}^{+5}\right)^{\circ}$, provided the first experimental evidence for non-axial character of SD structures around $A \approx 40$. The value obtained for the ground state, $\gamma=28(3)^{\circ}$, was interpreted as resulting from its softness.

This experimental work triggered new LSSM calculations for ${ }^{42} \mathrm{Ca}[3,19]$. They were performed using the SDPF.MIX interaction in the $\operatorname{sdpf}$ model space for neutrons and protons, with a virtual ${ }^{28} \mathrm{Si}$ core, as in the earlier work [21] that successfully described properties of the deformed $4 p-4 h$ and $8 p-8 h$ structures in ${ }^{40} \mathrm{Ca}$. Up to 6 particle-hole excitations from the $2 s_{1 / 2}$ and $1 d_{3 / 2}$ orbitals into the $p f$ orbitals were allowed, and the electric effective charges were $1.5 e$ for protons and $0.5 e$ for neutrons. The overall agreement of the calculations with the experimental level energies and decay patterns is remarkable. The experimental values of the $\left\langle Q^{2}\right\rangle$ and $\left\langle Q^{3} \cos 3 \delta\right\rangle$ invariants for the $0_{1,2}^{+}$states were also well reproduced. The only notable systematic difference is the overestimation of $E 2$ matrix elements in the SD band and underestimation of those in the yrast band as well as intra-band ones, which suggests that the mixing between the two bands is not fully reproduced by the calculations.

The LSSM results provide insight into the configurations of normal-deformed and SD states in ${ }^{42} \mathrm{Ca}$ : the $0_{2}^{+}$and $2_{2}^{+}$states are predicted to be dominated by the $6 p-4 h$ 
excitation, while the ground-state band has a predominantly two-particle configuration, with considerable $4 p-2 h$ and $6 p-4 h$ admixtures. Furthermore, they suggest that the experimentally known $2_{3}^{+}$state is the band head of a $K=2 \gamma$ band related to the SD structure, with the configuration dominated by almost equal contributions of $6 p-4 h$ and $8 p-6 h$ excitations $(\approx 40 \%$ each). This gives further support for the slightly triaxial shape of the SD band in ${ }^{42} \mathrm{Ca}$, while the identification of higher-spin members of the predicted $K=2 \gamma$ band represents a challenge for future experimental studies.

\subsection{Shape Coexistence, Triaxiality, and the $N=50$ Shell Closure in Germanium and Zinc Isotopes}

Detailed low-energy Coulomb-excitation studies were performed to investigate quadrupole properties of stable and exotic Ge and $\mathrm{Zn}$ isotopes, which are important in the context of the numerous Shell-Model calculations developed for this region. While extensive sets of electromagnetic matrix elements were extracted for the stable nuclei and interpreted within the quadrupole sum rules approach, in neutron-rich isotopes these measurements provided the first access to $B(E 2)$ values and, in some cases, also excitation energies.

In the stable Ge isotopes, the $\left\langle Q^{2}\right\rangle$ invariants extracted for the ground state and the $\mathrm{O}_{2}^{+}$state via low-energy Coulomb excitation represent one of the strongest signatures of shape coexistence $[22,23]$. As shown in Fig. $2 \mathrm{a}$, the ground-state $\left\langle Q^{2}\right\rangle$ values in ${ }^{70-76} \mathrm{Ge}$ are similar, $0.2-0.3 e^{2} \mathrm{~b}^{2}$, while those of the $0_{2}^{+}$states evolve as a function of the neutron number. The $0_{2}^{+}$state in ${ }^{70} \mathrm{Ge}$ is more deformed than the ground state [24], in ${ }^{72} \mathrm{Ge}$ both states seem to have comparable overall deformations and considerable triaxiality [25], while those for the $0_{2}^{+}$states in ${ }^{74,76} \mathrm{Ge}$ point to nearly spherical shapes [26,27]. Based on the similarity of the $0_{2}^{+}$energy systematics in Ge and $\mathrm{Zn}$ nuclei (see Fig. 2b), one could speculate that shape coexistence is present also in the latter isotopic chain. First hints of the intruder character of the $0_{2}^{+}$states in the $\mathrm{Zn}$ isotopes came from $E 0$ measurements in the stable even-even ${ }^{64-68} \mathrm{Zn}$ isotopes [28], a feature further supported by the results of multi-step Coulomb-excitation experiments on ${ }^{66,68} \mathrm{Zn}[15,29]$. However, only for ${ }^{68} \mathrm{Zn}$ has the key $\left\langle 2_{3}^{+}\|E 2\| 0_{2}^{+}\right\rangle$matrix element been determined which, when combined with other matrix elements involving the $0_{2}^{+}$state, leads to a $\left\langle Q^{2}\right\rangle$ invariant significantly different from that of the ground state [29]. On the other hand, multiple low-energy Coulomb-excitation studies of stable Ge and $\mathrm{Zn}$ isotopes [15,26,29] pointed to the importance of the triaxial degree of freedom in their structure, which was also evoked for the neighbouring ${ }^{76,78} \mathrm{Se}$ nuclei $[30,31]$. Particularly relevant is the work on ${ }^{76} \mathrm{Ge}[32]$ that yielded $\left(\beta_{2}, \gamma\right)$ parameters for the $0_{1}^{+}, 2_{1}^{+}$and $2_{2}^{+}$states and their dispersions, which are consistent with rigid triaxial deformation. This is particularly important considering that ${ }^{76} \mathrm{Ge}$ is a candidate for searches of neutrinoless double- $\beta$ decay, and the nuclear shape is predicted to play a significant role in this process $[33,34]$.

Shell-model calculations focusing on the Ge, $\mathrm{Zn}$, and Se isotopes reproduced well the features related to their triaxial shapes $[15,30,35,36]$, even though the degree of $\gamma$ softness and the presence of static triaxial deformation are still debated [35]. The V-shaped pattern of the $0_{2}^{+}$excitation energies in the Ge isotopes between $N=36$ and $N=44$ (see Fig. 2b) was related to shape coexistence by Shell-Model calculations [36] using the JUN45 effective interaction in a model space consisting of the ${ }^{56} \mathrm{Ni}$ inert core and up to the $1 g_{9 / 2}$ orbital for both neutrons and protons. The collectivity of the deformed ground states was linked to strong correlations (arising from pairing and the quadrupole-quadrupole force), which offset the $N=40$ gap and lead to the enhanced occupation of the $1 g_{9 / 2}$ neutron orbital that has a maximum predicted for $N=40$. In contrast, the role of neutron excitations from the $f p$ shell into the $1 g_{9 / 2}$ orbital is smaller for the $0_{2}^{+}$states, with, on average, two additional neutrons promoted through the $N=40$ gap with respect to the normal-order configuration. In particular, the wave function of the $0_{2}^{+}$state in ${ }^{72} \mathrm{Ge}$ is dominated by the normal-order configuration, i.e. neutrons completely filling the $f p$ shell, with a contribution of $37 \%$, which suggests a nearly spherical shape.

As shown in Fig. $2 b$, a decrease of the $0_{2}^{+}$state energy between $N=36$ and $N=40$, similar to those observed in the Ge and $\mathrm{Zn}$ chains, is evident also in the Ni isotopes. 

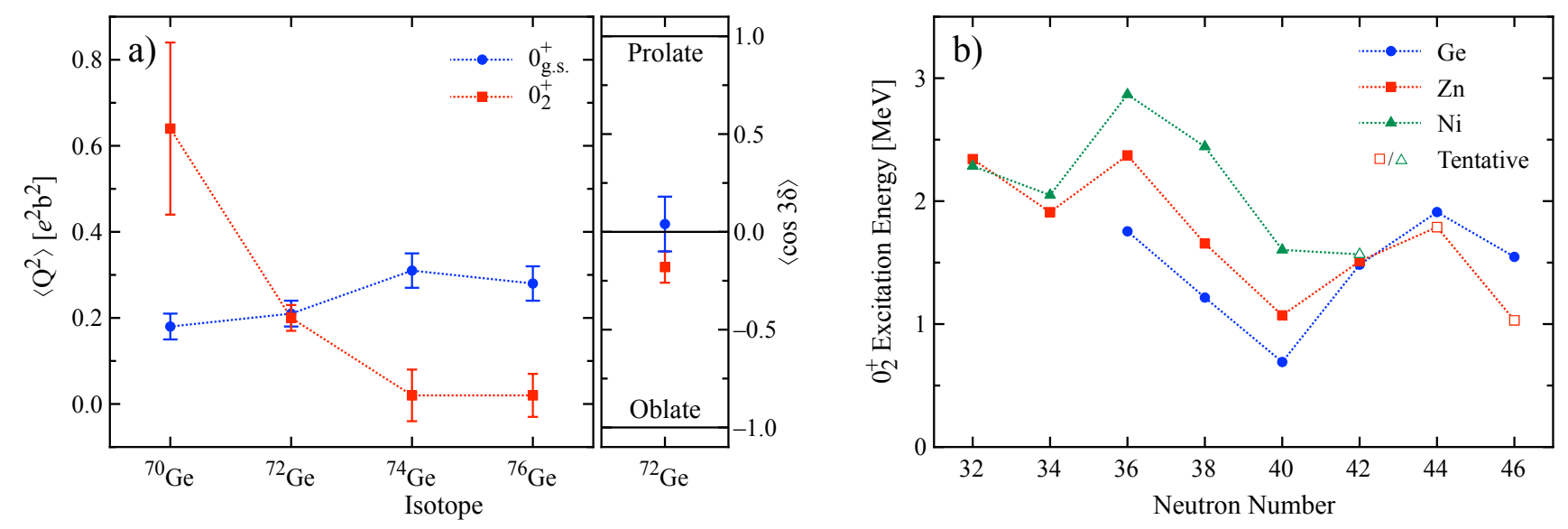

Figure 2. (Panel a) $\left\langle Q^{2}\right\rangle$ and $\langle\cos 3 \delta\rangle$ quantities for the $0_{\text {g.s. }}^{+}$and $0_{2}^{+}$states in the Ge isotopes. Data are taken from Refs. [22,24-27]. (Panel b) Systematics of excitation energies for $\mathrm{O}_{2}^{+}$states in $\mathrm{Ge}, \mathrm{Zn}$ and Ni isotopes from $N=32$ to $N=46$. Tentative spin assignments are shown with open symbols. Data are taken from the ENSDF database [37] and Refs. [38-40]. (Color online.)

According to MCSM calculations with the A3DA effective interaction in the $p f g_{9 / 2} d_{5 / 2}$ model space [41], the $0_{2}^{+}$states in ${ }^{64,66,68} \mathrm{Ni}$ are oblate deformed and result from neutron $2 p-2 h$ excitation across the $N=40$ gap, similar to their counterparts in the Ge isotones. The V-shaped trend of the $0_{2}^{+}$excitation energies with the vertex at $N=40$ does not persist for ${ }^{70} \mathrm{Ni}$ and beyond, as different configurations start to appear at low excitation energy. Specifically, proton $2 p-2 h$ excitations across the energy gap at $Z=28$ are suggested $[41,42]$ to dominate the structure of the $0_{4}^{+}$state in ${ }^{64,66} \mathrm{Ni}$, the $0_{3}^{+}$state in ${ }^{68} \mathrm{Ni}$ and the $0_{2}^{+}$state in ${ }^{70} \mathrm{Ni}$. MCSM calculations predict that these predominantly $\pi(2 p-2 h)$ states have well-deformed prolate shapes, resulting from an interplay of type-I and type-II shell evolution. The experimental verification of this multiple shape-coexistence scenario through the quadrupole sum rules approach represents a challenge for future low-energy Coulomb-excitation studies. Unfortunately, the population of excited $0^{+}$states in both stable and radioactive Ni nuclei will be severely limited due to the high excitation energies involved, which is further complicated by the prohibitively low intensities of radioactive $\mathrm{Ni}$ beams that are currently available at energies suitable for low-energy Coulomb excitation.

On the neutron-rich side, low-energy Coulomb excitation has provided valuable structure information in the Ge and $\mathrm{Zn}$ isotopes. Experiments at ISOLDE identified the first excited $2_{1}^{+}$state in ${ }^{78,80} \mathrm{Zn}$ and yielded the $B\left(E 2 ; 2_{1}^{+} \rightarrow 0_{g . s}^{+}\right)$values in ${ }^{74-80} \mathrm{Zn}$ and the $B\left(E 2 ; 4_{1}^{+} \rightarrow 2_{1}^{+}\right)$values in ${ }^{74,76} \mathrm{Zn}[43,44]$. The obtained $B(E 2)$ values hint at the importance of triaxiality also in neutron-rich $\mathrm{Zn}$ isotopes, whose ground states were suggested to be rather diffuse in the $\gamma$ degree of freedom [43]. Furthermore, the energy of the first excited state in ${ }^{80} \mathrm{Zn}$ confirms the persistence of the $N=50$ shell closure two protons away from the doubly-magic ${ }^{78} \mathrm{Ni}$. The same conclusion was reached for the neutron-rich Ge isotopes from the $B\left(E 2 ; 2_{1}^{+} \rightarrow 0_{g . s}^{+}\right)$values of the radioactive ${ }^{78,80}$ Ge measured using low-energy Coulomb excitation at ORNL [45]. The measured $B\left(E 2 ; 2_{1}^{+} \rightarrow 0_{1}^{+}\right)$values in ${ }^{74-80} \mathrm{Zn}$ were found in good agreement with those deduced from the experimental $2_{1}^{+}$excitation energies via the Grodzins rule, provided that a renormalization factor (0.92) was applied to the calculated values [43]. The experimental results for ${ }^{74-80} \mathrm{Zn}$ and ${ }^{78,80} \mathrm{Ge}$ were compared with Shell-Model calculations comprising the $2 p_{3 / 2}, 1 f_{5 / 2}, 2 p_{1 / 2}$, and $1 g_{9 / 2}$ orbitals for both protons and neutrons outside of an inert ${ }^{56} \mathrm{Ni}$ core. Effective charges significantly different from the standard $e_{v}=0.5 e, e_{\pi}=1.5 e$ values were adopted to compensate for the enhanced ${ }^{56} \mathrm{Ni}$ core polarization reported in Refs. [46,47]. The persistence of the $N=50$ shell closure in neutron-rich $\mathrm{Zn}$ and Ge isotopes, emerging from the experimental and calculated $B(E 2)$ values and excitation energies, anticipated the more recent results for ${ }^{78} \mathrm{Ni}$ in which the first excited $2_{1}^{+}$state was ultimately identified [48]. 


\subsection{Shape Coexistence in $\mathrm{Z} \approx 40 \mathrm{Nuclei}$}

The sudden onset of deformation at $N=60$ observed in the $\mathrm{Zr}$ and Sr isotopic chains has attracted a lot of attention, both from theoretical and experimental points of view. While the energies of the $2_{1}^{+}$states in ${ }^{90-100} \mathrm{Zr}$ were well reproduced by the LSSM calculations reported in Ref. [49], the required truncations of the model space made it impossible to account for the enhanced transition probability in ${ }^{100} \mathrm{Zr}$. Recently, the rapidity of the shape transition in the $\mathrm{Zr}$ isotopes has been reproduced, for the first time both in terms of level energies and transition probabilities, using the MCSM [50]. The calculations also predict that ${ }^{94,96,98,100} \mathrm{Zr}$ would present a multitude of low-lying states with various quadrupole shapes. A Coulomb-excitation study of ${ }^{94} \mathrm{Zr}$ aiming to verify this scenario has been performed at INFN-LNL [51] and its analysis is in progress. There exists, however, strong experimental evidence for the coexistence of deformed and spherical structures in ${ }^{96,98} \mathrm{Sr}$, recently reinforced by the results of Coulomb-excitation experiments performed at ISOLDE [2,52]. The rich set of transitional and diagonal $E 2$ matrix elements determined in this study provides a consistent picture of a prolate-deformed ground-state band in ${ }^{98} \mathrm{Sr}$ that coexists with an almost spherical structure built on the $0_{2}^{+}$state. Similarity of the $B\left(E 2 ; 2_{2}^{+} \rightarrow 0_{2}^{+}\right)=13(2)$ W.u. value in ${ }^{98} \mathrm{Sr}$ with the $B\left(E 2 ; 2_{1}^{+} \rightarrow 0_{1}^{+}\right)=17_{-3}^{+4}$ W.u. value in ${ }^{96} \mathrm{Sr}$, as well as of the quadrupole moments of the $2_{2}^{+}$state in ${ }^{98} \mathrm{Sr}$ and the $2_{1}^{+}$state in ${ }^{96} \mathrm{Sr}$ (both compatible with zero), suggest that the spherical and deformed structures interchange at $N=60$. Also, contrary to what is observed in most known cases of shape coexistence, these two structures mix very weakly. This feature is in line with the type-II shell evolution scenario proposed by Ref. [50] that links particular multiparticle-multihole excitations to significant reorganisations of the shell structure, which hinders configuration mixing.

A notable result of Refs. [2,52] is the observed reduction of the $Q_{s}\left(2_{1}^{+}\right)$value in ${ }^{98} \mathrm{Sr}$ with respect to the rotational estimate. This feature may indicate triaxiality of this state, which gives way to a more prolate deformation for higher-spin members of the groundstate band. Detailed Coulomb-excitation studies of ${ }^{96,98,100}$ Mo $[18,53]$ yielded $\left\langle Q^{2}\right\rangle$ and $\left\langle Q^{3} \cos 3 \delta\right\rangle$ invariants for the ground states and the low-lying $0_{2}^{+}$states, demonstrating their different shapes and confirming that triaxiality is also a key feature of Mo nuclei with $A \approx 100$. The obtained invariant quantities indicate that in ${ }^{96} \mathrm{Mo}$ an almost spherical $\mathrm{O}_{2}^{+}$ state coexists with a triaxial ground state, while in ${ }^{98} \mathrm{Mo}$ both the $\mathrm{O}_{1}^{+}$and $\mathrm{O}_{2}^{+}$states have approximately the same values of $\left\langle Q^{2}\right\rangle$. However, the $\langle\cos 3 \delta\rangle$ values suggest that the ground state in ${ }^{98} \mathrm{Mo}$ is triaxial and the $0_{2}^{+}$state has a prolate shape. The same pattern of a prolate $0_{2}^{+}$state coexisting with a triaxial ground state appears in ${ }^{100} \mathrm{Mo}$, but the $\left\langle Q^{2}\right\rangle$ invariants obtained for both the $0_{1}^{+}$and $0_{2}^{+}$states in this nucleus are significantly greater than those for ${ }^{98} \mathrm{Mo}$, with that for the $0_{2}^{+}$state being much larger. Given also that the proton vacancies and neutron occupancies for the ground states of ${ }^{98,100}$ Mo were recently extracted from an extensive series of single-proton and single-neutron transfer reactions [54], these nuclei would represent a stringent test for Shell-Model calculations.

\subsection{Evolution of Collectivity in $\mathrm{Z} \approx 50 \mathrm{Nuclei}$}

The tin nuclei, forming the longest chain of experimentally accessible isotopes between two doubly-magic nuclei, have traditionally been considered a prime example of seniority scheme. While this description is supported by the almost constant energies of the $2_{1}^{+}$states in the even-even $\mathrm{Sn}$ nuclei from ${ }^{102} \mathrm{Sn}$ to ${ }^{130} \mathrm{Sn}$, the corresponding $B\left(E 2 ; 2_{1}^{+} \rightarrow 0_{1}^{+}\right)$values seem to deviate from the expected parabolic behaviour. Extensive Coulomb-excitation studies of stable [55-57] and exotic Sn nuclei [58-62] yielded $B\left(E 2 ; 2_{1}^{+} \rightarrow 0_{1}^{+}\right)$values for ${ }^{106-134} \mathrm{~S} n$ that were discussed in the context of Shell-Model calculations.

In the Coulomb-excitation campaigns aiming at high-precision measurements of the $B\left(E 2 ; 2_{1}^{+} \rightarrow 0_{1}^{+}\right)$values in stable $\mathrm{Sn}$ isotopes, the experimental conditions minimised the role of multi-step excitation and the reorientation effect. The experiments at ORNL [55] were performed in strongly inverse kinematics, with a ${ }^{12} \mathrm{C}$ target bombarded by $112,114,116,118,120,122,12$ beams; a ${ }^{\text {nat }} \mathrm{Ti}$ target was also used for complementary $Q_{s}\left(2_{1}^{+}\right)$measurements. In the IUAC 
campaign [56,57], a reaction partner with a much higher $Z$ was used: a ${ }^{58} \mathrm{Ni}$ beam impinged on ${ }^{112,116,118,120,122,124} \mathrm{Sn}$ targets. However, due to the selection of events with the $\mathrm{Ni}$ beam particles scattered at forward angles, no excitation of higher-lying states was observed, although their possible weak influence on the $2_{1}^{+}$excitation process was taken into account in the data analysis. The $B\left(E 2 ; 2_{1}^{+} \rightarrow 0_{1}^{+}\right)$values were obtained with relative uncertainties of $5 \%$ or less in all cases, and the results of the two campaigns agreed within $3 \sigma$ for ${ }^{120,122,124} \mathrm{Sn}$ and within $1 \sigma$ for the other isotopes demonstrating the level of accuracy and precision that can be achieved.

Low-energy Coulomb-excitation experiments on neutron-deficient $\mathrm{Sn}$ isotopes were performed at ISOLDE $[58,59]$ with $2.8-\mathrm{MeV} / \mathrm{A}{ }^{106,108,110} \mathrm{Sn}$ beams bombarding ${ }^{58} \mathrm{Ni}$ targets. On the neutron-rich side, a campaign was performed at ONRL $[60,61]$ to study $126,128,130,134 \mathrm{Sn}$ in very similar experimental conditions as those used for stable isotopes in Ref. [55]. In order to increase the excitation cross section for the $2_{1}^{+}$state in ${ }^{132} \mathrm{Sn}$, located at 4.04-MeV excitation energy, targets of ${ }^{48} \mathrm{Ti}$ and ${ }^{206} \mathrm{~Pb}$ were used in the ONRL [60] and HIE-ISOLDE [62] measurements, respectively.

While certain discrepancies with the values obtained using other methods exist (see e.g. Ref. [63] for a compilation of experimental data), the ensemble of experimental results points to an asymmetric shape of the $B\left(E 2 ; 2_{1}^{+} \rightarrow 0_{1}^{+}\right)$distribution as a function of $N$, with a plateau extending towards lighter nuclei. The reproduction of this plateau represented a challenge for model calculations. Recently, its appearance has been discussed $[64,65]$ in the context of pseudo-SU(3) symmetry acting in the space of $g d s$ orbitals excluding $1 g_{9 / 2}$. The calculations were performed using $V_{\text {low }-k}$ variants of the realistic N3LO interaction, with the monopole part of the interaction replaced by a Hamiltonian provided by the GEMO code [66], adding the single-particle energies for ${ }^{101} \mathrm{Sn}$. They successfully reproduced the evolution of the $B\left(E 2 ; 2_{1}^{+} \rightarrow 0_{1}^{+}\right)$values in ${ }^{104-114} \mathrm{Sn}[64,65]$ and shown that modifications of the pairing strength had a negligible effect on the calculated $B\left(E 2 ; 2_{1}^{+} \rightarrow 0_{1}^{+}\right)$values, in contrast to what was observed for the $B\left(E 2 ; 4_{1}^{+} \rightarrow 2_{1}^{+}\right)$strengths [65]. An alternative explanation was offered by MCSM calculations [63] performed in the full $g d s$ model space complemented by the $1 h_{11 / 2}, 2 f_{7 / 2}$, and $3 p_{3 / 2}$ orbitals for protons and neutrons. The calculations of Ref. [63] provide good reproduction of all measured $B\left(E 2 ; 2_{1}^{+} \rightarrow 0_{1}^{+}\right)$values in the Sn chain, including the local increase observed for ${ }^{132} \mathrm{Sn}$, and link their enhancement for ${ }^{108-114} \mathrm{~S} n$ to the development of quadrupole deformation driven by proton excitations from the $1 g_{9 / 2}$ orbital. This scenario is consistent with the observed increase of the $Q_{s}\left(2_{1}^{+}\right)$ values at mid shell [55], which was suggested to be due to the mixing with a deformed configuration, resulting in the presence of proton $2 p-2 h$ and $4 p-4 h$ components in the $2_{1}^{+}$ wave function [55]. Low-lying states of predominantly proton $2 p-2 h$ character have been identified in ${ }^{114,116,118} \mathrm{Sn}$ via two-proton transfer reactions [67], and later also in ${ }^{110,112} \mathrm{Sn}$ and ${ }^{120,122,124} \mathrm{Sn}$, although at higher excitation energies. Calculations of Ref. [63] predict indeed that the ground states of $\mathrm{Sn}$ nuclei involve a significant promotion of protons across the $Z=50$ gap, with the largest $2 d_{5 / 2}$ occupation predicted at $N=60$. The occupation of proton orbitals above the $Z=50$ gap becomes even larger for the $2_{1}^{+}$states, and the corresponding T-plots indicate deformed shapes [63], in line with the measured non-zero quadrupole moments. Multi-step Coulomb-excitation studies aiming at the determination of deformation parameters of the deformed structures built on the $0_{2}^{+}$states, as well as their mixing with the ground-state configurations, would be of much interest. One should note here that quadrupole invariants for the $0_{1,2}^{+}$states in ${ }^{110} \mathrm{Cd}$ were measured in a recent Coulomb-excitation experiment [68].

The $B\left(E 2 ; 2_{1}^{+} \rightarrow 0_{1}^{+}\right)$and $B\left(E 2 ; 4_{1}^{+} \rightarrow 2_{1}^{+}\right)$patterns in ${ }^{100-110} C d$ nuclei closely resemble that of the $B\left(E 2 ; 2_{1}^{+} \rightarrow 0_{1}^{+}\right)$values in the corresponding $S n$ isotones. They were well reproduced by the calculation of Ref. [65], and found almost independent of the assumed pairing strength. This was linked [65] to their static quadrupole deformation, consistent with non-zero quadrupole moments measured for ${ }^{102,104} \mathrm{Cd}$ isotopes in a Coulomb-excitation experiment at ISOLDE [69]. Interestingly, the obtained $Q_{s}\left(2_{1}^{+}\right)$values are positive, in contrast to those measured for stable Cd nuclei. Unfortunately, they are subject to large un- 

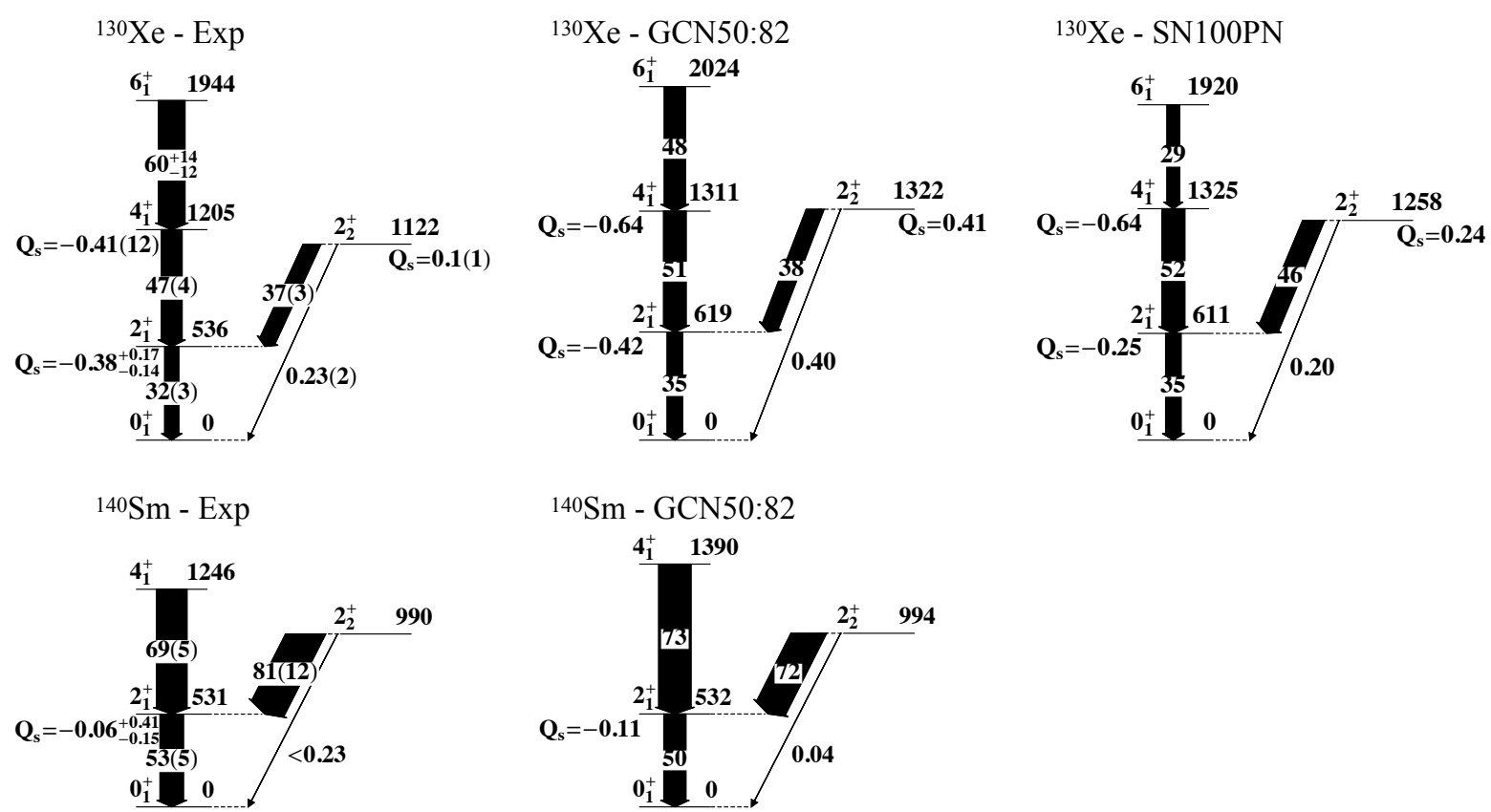

Figure 3. Comparison of low-energy parts of the experimental ${ }^{130} \mathrm{Xe}$ and ${ }^{140} \mathrm{Sm}$ level schemes with Shell-Model calculations [17,72]. The states are labelled with their spin and parity $I^{\pi}$ and excitation energy in keV. Transitions are labelled with reduced transition probabilities expressed in Weisskopf units. Spectroscopic quadrupole moments are reported in $e$ b. See the text for further details about the calculations.

certainties, and the $Q_{s}\left(2_{1}^{+}\right)$value for ${ }^{104} \mathrm{Cd}$ significantly changes if a previously measured lifetime of the $2_{1}^{+}$state is used as an additional constraint in the Coulomb-excitation data analysis.

Quadrupole deformation of light Cd isotopes was explored in an LSSM study [16] using a modified $v 3 s b$ effective interaction [70] in the $\pi\left(2 p_{1 / 2}, 1 g_{9 / 2}\right), v\left(2 d_{5 / 2}, 3 s_{1 / 2}, 2 d_{3 / 2}\right.$, $\left.1 g_{7 / 2}, 1 h_{11 / 2}\right)$ model space. The calculated $E 2$ matrix elements provide a good reproduction of the experimental $B\left(E 2 ; 2_{1}^{+} \rightarrow 0_{1}^{+}\right)$and $B\left(E 2 ; 4_{1}^{+} \rightarrow 2_{1}^{+}\right)$values, and were analysed in terms of quadrupole invariants $\left\langle Q^{2}\right\rangle$ and $\left\langle Q^{3} \cos 3 \delta\right\rangle$ pointing to a predominantly prolate character of ${ }^{100-108} \mathrm{Cd}$ with both $\beta$ and $\gamma$ increasing with $N$. Very recently, Coulomb excitation of ${ }^{106} \mathrm{Cd}$ was performed [71] at the NSCL ReA3 facility. Quadrupole moments of the $2_{1}^{+}, 4_{1}^{+}, 6_{1}^{+}$and $2_{2}^{+}$states were obtained, as well as the $\left\langle Q^{2}\right\rangle$ and $\left\langle Q^{3} \cos 3 \delta\right\rangle$ invariants for the ground state, which suggest its considerable triaxiality. This feature does not emerge from the LSSM calculations reported in Ref. [71], which also used a G-matrixrenormalized CD-Bonn nucleon-nucleon potential and the same model space as those of Ref. [16], but allowed at most two neutrons in the $1 h_{11 / 2}$ orbital. While they well reproduced the experimental $\left\langle Q^{2}\right\rangle$ invariant, the shapes that they predict for light $\mathrm{Cd}$ isotopes are decidedly prolate. The difference with respect to a more $\gamma$-soft behaviour suggested by Ref. [16] was attributed to the different $1 h_{11 / 2}$ single-particle energies, as well as the adopted truncation. However, none of these calculations are able to explain the observed pattern of spectroscopic quadrupole moments in the light $\mathrm{Cd}$ nuclei, which hopefully will trigger more experimental and theoretical work aiming at understanding their quadrupole properties.

\subsection{Heavier Collective Nuclei: Triaxiality in ${ }^{130} \mathrm{Xe}$ and ${ }^{140} \mathrm{Sm}$}

The ${ }^{130} \mathrm{Xe}$ and ${ }^{140} \mathrm{Sm}$ isotopes are examples of relatively heavy nuclei, probed with low-energy Coulomb excitation, for which extensive Shell-Model calculations have been performed [17,72]. Both isotopes were studied at ISOLDE, with the measurement for the stable ${ }^{130} \mathrm{Xe}$ being a by-product of a radioactive beam experiment. Beam energies were $4.2 \mathrm{MeV} / \mathrm{u}$ and $2.8 \mathrm{MeV} / \mathrm{u}$, respectively, and states up to $I^{\pi}=6_{1}^{+}$were observed in ${ }^{130} \mathrm{Xe}$, 
while the $2_{1}^{+}, 4_{1}^{+}$and $2_{2}^{+}$states were populated in ${ }^{140} \mathrm{Sm}$. The results point to the importance of the triaxial degree of freedom in the structure of low-lying levels in both nuclei. The extracted transitional and diagonal E2 matrix elements indicate that ${ }^{130} \mathrm{Xe}$ and ${ }^{140} \mathrm{Sm}$ are collective and their ground states are characterized by $\beta_{2} \approx 0.15$ and $\gamma \approx 30^{\circ}$. For ${ }^{130} \mathrm{Xe}$, this conclusion was drawn on the basis of the determined quadrupole invariants, while for ${ }^{140} \mathrm{Sm}$ it results from the measured $Q_{s}\left(2_{1}^{+}\right)=-0.06_{-0.15}^{+0.41} \mathrm{eb}$, compatible with zero, and the enhanced $B\left(E 2 ; 2_{1}^{+} \rightarrow 0_{\text {g.s. }}^{+}\right)=53$ (5) W.u. value. Shell-Model calculations for ${ }^{130} \mathrm{Xe}$ and ${ }^{140} \mathrm{Sm}$ were performed in a large model space consisting of the ${ }^{100} \mathrm{Sn}$ inert core and all orbitals up to $N=Z=82$. The GCN50:82 effective interaction [73] was employed for both cases, complemented by the SN100PN effective interaction [74] for ${ }^{130} \mathrm{Xe}$. The experimental and theoretical results show a good agreement (see Figure 3), which is remarkable considering the evident collective nature of the two nuclei and the relatively high number of allowed valence particles in the Shell-Model calculations. However, for both ${ }^{130} \mathrm{Xe}$ and ${ }^{140} \mathrm{Sm}$, effective charges larger than the standard $e_{v}=0.5 e, e_{\pi}=1.5 e$ values were needed to reproduce the measured $B(E 2)$ values. For ${ }^{130} \mathrm{Xe}, e_{v}=0.945 e, e_{\pi}=1.53 e$ and $e_{v}=0.84 e, e_{\pi}=1.68 e$ were adopted for the GCN50:82 and SN100PN interactions, respectively, while $e_{v}=0.64 e, e_{\pi}=1.65 e$ were used for the GCN50:82 interaction in the case of ${ }^{140} \mathrm{Sm}$. The need for increasing the effective charges in this mass region with respect to the standard values is known $[75,76]$, and suggests that a further expansion of the model space is necessary.

Despite the good reproduction of the experimental results by state-of-the-art ShellModel calculations, further developments are needed to properly describe the structure of $A \approx 130-140$ nuclei within this theoretical approach. This is particularly relevant for ${ }^{130} \mathrm{Xe}$, which would be the daughter of the ${ }^{130} \mathrm{Te}$ neutrinoless double- $\beta$ decay. If this process is observed at ongoing experiments, such as CUORE [77] and SNO+ [78], the relevant $\beta \beta$ nuclear matrix elements will need to be calculated in order to extract the Majorana mass. Such calculations are under way, also within the Shell Model [79]. Further low-energy Coulomb-excitation studies should help to elucidate the nuclear structure at $A \approx 130-140$. A ${ }^{130}$ Xe beam could be delivered by a stable ion beam facility with a much higher intensity than that available in Ref. [17], and the use of a heavier target (e.g. ${ }^{208} \mathrm{~Pb}$ ) would increase the excitation cross sections. For ${ }^{140} \mathrm{Sm}$, an experiment with a higher beam energy would be beneficial. Under favourable conditions, such experiments should be capable of extracting higher-order quadrupole invariants related to the dispersions in $\beta_{2}$ and $\gamma$ for the ground state.

\section{Summary and Outlook}

In parallel to recent technological advances in accelerator and ion-source technologies, and the construction of new-generation high-resolution $\gamma$-ray tracking arrays as AGATA [20] and GRETINA [80], noteworthy developments have taken place in nuclearstructure theory. The state-of-the-art calculations, some of which were discussed in the preceding sections, are now able to predict properties of nuclei with an unprecedented level of detail, particularly concerning the nuclear shape. Within the Shell Model, T-plots [41] and methods based on the use of the projected Lanczos strength function [35] describe the nuclear shape for both ground and excited states. These ongoing experimental and theoretical developments will bring forward our understanding of nuclear structure, while also being relevant for cross-disciplinary fields such as astrophysics, neutrino physics, and physics of (and beyond) the Standard Model $[4,34,81]$. In this context, a precise understanding of the nuclear shape can bring us closer to answering long-standing questions in physics, such as how heavy elements originate in cataclysmic stellar events and the reason for the matter-antimatter asymmetry in the Universe.

Thanks to the constant development of powerful computational resources, and refinements of Shell-Model codes and methods, this theoretical approach can now be extended to vast regions of the nuclear chart. It can be anticipated that this progress will be complemented and inspired by the availability of high-precision spectroscopic data and that 
CUORE

ENSDF

GRETINA HIE-ISOLDE

INFN

ISOL

ISOLDE

IUAC

501

LNL

low-energy Coulomb excitation will continue to play an important role in future studies throughout the nuclear chart. We emphasize, however, as in the case of ${ }^{98,100} \mathrm{Mo}$ that the combination of data from a variety of techniques that probe both collective and singleparticle degrees of freedom will provide perhaps the most demanding tests of Shell-Model

Funding: This research was funded in part through the Natural Sciences and Engineering Research

Acknowledgments: We thankfully acknowledge P.E. Garrett for fruitful discussions and for the careful reading of the manuscript.

Conflicts of Interest: The authors declare no conflict of interest.

\section{Abbreviations}

he following abbreviations are used in this manuscript:

AGATA Advanced GAmma Tracking Array

LSSM

MCSM

Cryogenic Underground Laboratory for Rare Events

Evaluated Nuclear Structure Data File

Gamma-Ray Energy Tracking In-beam Nuclear Array

NSCL

ORNL

High Intensity and Energy ISOLDE

Istituto Nazionale di Fisica Nucleare (National Institute for Nuclear Physics)

ReA3

Isotope Separation On-Line

Isotope Separator On-Line DEvice

Inter-University Accelerator Centre

Legnaro National Laboratories

Large-Scale Shell Model

Monte-Carlo Shell Model

National Superconducting Cyclotron Laboratory

RIB

Oak Ridge National Laboratory

$\mathrm{SD}$

Re-accelerator facility

Radioactive Ion Beam

T-Plot

Superdeformed

Sudbury Neutrino Observatory Plus

Tsunoda-Plot

\section{References}

1. Kumar, K. Intrinsic quadrupole moments and shapes of nuclear ground states and excited states. Phys. Rev. Lett. 1972, $28,249$.

2. Clément, E.; Zielińska, M.; Görgen, A.; et al. Spectroscopic quadrupole moments in ${ }^{96,98}$ Sr: evidence for shape coexistence in neutron-rich strontium isotopes at $N=60$. Phys. Rev. Lett. 2016, 116, 022701.

3. Hadyńska-Klęk, K.; Napiorkowski, P.J.; Zielińska, M.; et al. Superdeformed and triaxial states in ${ }^{42}$ Ca. Phys. Rev. Lett. 2016, 117, 062501.

4. Gaffney, L.P.; Butler, P.A.; Scheck, M.; et al. Studies of pear shaped nuclei using accelerated radioactive beams. Nature 2013, 497, 199.

5. Zielińska, M.; Gaffney, L.P.; Wrzosek-Lipska, K.; et al. Analysis methods of safe Coulomb-excitation experiments with radioactive ion beams using the GOSIA code. Eur. Phys. J. A 2016, 52, 99.

6. Görgen, A.; Korten, W. Coulomb excitation studies of shape coexistence in atomic nuclei. J. Phys. G: Nucl. Part. Phys. 2010, 43, 024002 .

7. Cline, D. Nuclear shapes studied by Coulomb excitation. Annu. Rev. Nucl. Part. Sci. 1986, 36, 683.

8. Srebrny, J.; Czosnyka, T.; Karczmarczyk, W.; et al. E1, E2, E3 and M1 information from heavy ion Coulomb excitation. Nucl. Phys. A 1993, 557, 663c.

9. Alder, K.; A. Winther, A. Electromagnetic Excitation; North-Holland: Amsterdam, Netherlands, 1975.

10. Nara Singh, B.S.; Nanal, V.; Pillay, R.G. Hexadecapole deformation studies in ${ }^{148,150}$ Nd. Pramana 2003, 61, 507.

11. De Boer, J.; Eichler, J. The Reorientation Effect, Advanced in nuclear physics; Springer: US, 1968; Vol. 1, chapt. 1, pp. 1-65.

12. Clément, E.; Görgen, A.; Korten, W.; et al. Shape coexistence in neutron-deficient krypton isotopes. Phys. Rev. C. 2007, 75, 054313.

13. Zielińska, M.; Hadyńska-Klęk, K. Nuclear shapes studied with low-energy Coulomb excitation. EPJ Web of Conf. 2018, 178, 02014.

14. Henderson, J. Convergence of electric quadrupole rotational invariants from the nuclear shell model. Phys. Rev. C 2020, 102, 054306 . 
15. Rocchini, M.; Hadyńska-Klęk, K.; Nannini A.; et al. Onset of triaxial deformation in ${ }^{66} \mathrm{Zn}$ and properties of its first excited $0^{+}$ state studied by means of Coulomb excitation. Phys. Rev. C 2021, 103, 014311.

16. Schmidt, T.; Heyde, K.L.G.; Blazhev, A.; Jolie, J. Shell-model-based deformation analysis of light cadmium isotopes. Phys. Rev. C 2017, 94, 014302.

17. Morrison, L.; Hadyńska-Klęk, K.; Podolyák, Zs.; et al. Quadrupole deformation of ${ }^{130}$ Xe measured in a Coulomb-excitation experiment. Phys. Rev. C 2020, 102, 054304.

18. Wrzosek-Lipska, K.; Próchniak, L.; Zielińska, M.; et al. Electromagnetic properties of ${ }^{100}$ Mo: experimental results and theoretical description of quadrupole degrees of freedom. Phys. Rev. C 2012, 86, 064305.

19. Hadyńska-Klęk, K.; Napiorkowski, P.J.; Zielińska, M.; et al. Quadrupole collectivity in ${ }^{42}$ Ca from low-energy Coulomb excitation with AGATA. Phys. Rev. C 2018, 97, 024326.

20. Akkoyun S.; Algora A.; Alikhani B.; et al. AGATA - Advanced GAmma Tracking Array. Nucl. Instrum. Methods Phys. Res. A 2012, $668,26$.

21. Caurier, E.; Menéndez, J.; Nowacki, F.; Poves, A. Coexistence of spherical states with deformed and superdeformed bands in doubly magic ${ }^{40} \mathrm{Ca}$ : A shell-model challenge. Phys. Rev. C 2007, 75, 054317.

22. Toh Y.; Czosnyka T.; Oshima M.; et al. Shape coexistence in even-even Ge isotopes - complete spectroscopy with Coulomb excitation. Journal of Nuclear Science and Technology 2002, 39, 497.

23. Heyde K.; Wood J.L. Shape coexistence in atomic nuclei. Rev. Mod. Phys. 2011, 83, 1467.

24. Sugawara, M.; Toh, Y.; Czosnyka, T.; et al. Multiple Coulomb excitation of a ${ }^{70} \mathrm{Ge}$ beam and the interpretation of the $0_{2}^{+}$state as a deformed intruder. Eur. Phys. J. A 2003, 16, 409.

25. Ayangeakaa, A.D.; Janssens, R.V.F.; Wu, C.Y.; et al. Shape coexistence and the role of axial asymmetry in ${ }^{72}$ Ge. Phys. Lett. B 2016, 754, 254.

26. Toh, Y.; Czosnyka, T.; Oshima, M.; et al. Coulomb excitation of ${ }^{74}$ Ge beam. Eur. Phys. J. A 2000, 9, 353.

27. Toh, Y.; Czosnyka, T.; Oshima, M.; et al. Multiple Coulomb excitation of a ${ }^{76}$ Ge beam J. Phys. G: Nucl. Part. Phys. 2001, $27,1475$.

28. Passoja, A.; Julin, R.; Kantele, J.; et al. E0 transitions in ${ }^{70} \mathrm{Ge}$ and shape-coexistence interpretation of even-mass Ge isotopes. Nucl. Phys. A 1985, 438, 413 .

29. Koizumi, M.; Seki, A.; Toh, Y.; et al. Multiple Coulomb excitation experiment of ${ }^{68}$ Zn. Nucl. Phys. A 2004, 730, 46.

30. Henderson J.; Wu C.Y.; Ash J.; et al. Triaxiality in selenium-76. Phys. Rev. C 2019, 99, 054313.

31. Hayakawa, T.; Toh, Y.; Oshima, M.; et al. Projectile Coulomb excitation of ${ }^{78}$ Se. Phys. Rev. C 2003, $67,064310$.

32. Ayangeakaa, A.D.; Janssens, R.V.F.; Zhu, S.; et al. Evidence for rigid triaxial deformation in ${ }^{76}$ Ge from a model-independent analysis. Phys. Rev. Lett. 2019, 123, 102501.

33. Caurier E.; Nowacki F.; Poves A. Nuclear-structure aspects of the neutrinoless $\beta \beta$-decays. Eur. Phys. J. A 2008, $36,195$.

34. Rodríguez, T.R.; Martínez-Pinedo, G. Energy density functional study of nuclear matrix elements for neutrinoless $\beta-\beta$ decay. Phys. Rev. Lett. 2010, 105, 252503.

35. Poves, A.; Nowacki, F.; Alhassid, Y. Limits on assigning a shape to a nucleus. Phys. Rev. C 2020, 101, 054307.

36. Honma, M.; Otsuka, T.; Mizusaki, T.; Hjorth-Jensen, M. New effective interaction for $f_{5} p g_{9}$-shell nuclei. Phys. Rev. C 2009, 80, 064323.

37. ENSDF, NNDC, Brookhaven National Laboratory, https://www.nndc.bnl.gov/ensdf/

38. Marginean, N.; Little, D.; Tsunoda, Y.; et al. Shape coexistence at zero spin in ${ }^{64} \mathrm{Ni}$ driven by the monopole tensor interaction. Phys. Rev. Lett. 2020, 125, 102502.

39. Tracy Jr., J.L.; Winger, J.A.; Rasco, B.C.; et al. Updated $\beta$-decay measurement of neutron-rich ${ }^{74}$ Cu. Phys. Rev. C 2018, $98,034309$.

40. Recchia, F.; Chiara, C.J.; Janssens, R.V.F.; et al. Configuration mixing and relative transition rates between low-spin states in ${ }^{68} \mathrm{Ni}$. Phys. Rev. C 2013, 88, 041302(R).

41. Tsunoda, Y.; Otsuka, T.; Shimizu, N.; Honma, M.; Utsuno Y. Novel shape evolution in exotic Ni isotopes and configurationdependent shell structure. Phys. Rev. C 2014, 89, 031301(R).

42. Leoni S.; Fornal B.; Marginean N.; et al. Shape coexistence and shape isomerism in the Ni isotopic chain. Acta Phys. Pol. B 2019, 50 605.

43. Van de Walle, J.; Aksouh, F.; Behrens, T.; et al. Low-energy Coulomb excitation of neutron-rich zinc isotopes. Phys. Rev. C 2009, 79, 014309.

44. Van de Walle, J.; Aksouh, F.; Ames, F.; et al. Coulomb excitation of neutron-rich Zn isotopes: First observation of the $2_{1}^{+}$state in ${ }^{80}$ Zn. Phys. Rev. Lett. 2007, 99, 142501.

45. Padilla-Rodal, E.; Galindo-Uribarri, A.; Baktash C.; et al. B(E2) $\uparrow$ measurements for radioactive neutron-rich Ge isotopes: Reaching the $N=50$ closed shell Phys. Rev. Lett. 2005, 94, 122501.

46. Honma, M.; Otsuka, T.; Brown, B.A.; Mizusaki, T. New effective interaction for $p f$-shell nuclei and its implications for the stability of the $N=Z=28$ closed core. Phys. Rev. C 2004, 69, 034335.

47. Otsuka, T.; Honma, M.; Mizusaki, T. Structure of the $N=Z=28$ closed shell studied by Monte Carlo Shell Model calculation. Phys. Rev. Lett. 1998, 81, 1588.

48. Taniuchi, R.; Santamaria, C.; Doornenbal, P.; et al. ${ }^{78} \mathrm{Ni}$ revealed as a doubly magic stronghold against nuclear deformation. Nature 2019, 569, 52. 
49. Sieja, K.; Nowacki, F.; Langanke, K.; Martínez-Pinedo, G. Shell model description of zirconium isotopes. Phys. Rev. C 2009, 79, 064310.

50. Togashi, T.; Tsunoda, Y.; Otsuka, T.; Shimizu, N. Quantum Phase Transition in the shape of Zr isotopes. Phys. Rev. Lett. 2016, 117, 172502.

51. Marchini, N.; Rocchini, M.; Nannini, A.; et al. Shape coexistence in ${ }^{94}$ Zr studied via Coulomb excitation. Eur. Phys. J Web of Conf. 2019, 223, 01038.

52. Clément, E.; Zielińska, M.; Péru, S.; et al. Low-energy Coulomb excitation of ${ }^{96,98}$ Sr beams. Phys. Rev. C 2016, $94,054326$.

53. Zielińska, M.; Czosnyka, T.; Choiński, J.; et al. Electromagnetic structure of ${ }^{98}$ Mo. Nucl. Phys. A 2002, $712,3$.

54. Freeman, S.J.; Sharp, D.K.; McAllister, S.A.; et al. Experimental study of the rearrangements of valence protons and neutrons amongst single-particle orbits during double- $\beta$ decay in ${ }^{100}$ Mo. Phys. Rev. C 2017, 96, 054325.

55. Allmond, J.M.; Stuchbery, A.E.; Galindo-Uribarri, A.; et al. Investigation into the semimagic nature of the tin isotopes through electromagnetic moments. Phys. Rev. C 2015, 92, 041303(R).

56. Kumar, R.; Saxena, M.; Doornenbal, P.; et al. No evidence of reduced collectivity in Coulomb-excited Sn isotopes. Phys. Rev. C 2017, 96, 054318.

57. Kumar, R.; Doornenbal, P.; Jhingan, A.; et al. Enhanced $0_{\text {g.s. }}^{+} \rightarrow 2_{1}^{+}$E2 transition strength in ${ }^{112}$ Sn. Phys. Rev. C 2010, $81,024306$.

58. Cederkäll, J.; Ekström, A.; Fahlander, C.; et al. Sub-barrier Coulomb excitation of ${ }^{110} \mathrm{Sn}$ and its implications for the ${ }^{100} \mathrm{Sn}$ shell closure. Phys. Rev. Lett. 2007, 98, 172501.

59. Ekström, A.; Cederkäll, J.; Fahlander, C.; et al. $0_{\text {g.s. }}^{+} \rightarrow 2_{1}^{+}$transition strengths in ${ }^{106}$ Sn and ${ }^{108}$ Sn. Phys. Rev. Lett. 2008, 101, 012502.

60. Radford, D.C.; Baktash, C.; Beene, J.R.; et al. Nuclear structure studies with heavy neutron-rich RIBS at the HRIBF. Nucl. Phys. A 2004, 746, 83c.

61. Allmond, J.M.; Radford, D.C.; Baktash, C.; et al. Coulomb excitation of ${ }^{124,126,128}$ Sn. Phys. Rev. C 2012, 84, 061303(R).

62. Rosiak, D.; Seidlitz, M.; Reiter, P.; et al. Enhanced quadrupole and octupole strength in doubly magic ${ }^{132}$ Sn. Phys. Rev. Lett. 2018, 121, 252501.

63. Togashi, T.; Tsunoda, Y.; Otsuka, T.; et al. Novel shape evolution in Sn isotopes from magic numbers 50 to 82 Phys. Rev. Lett. 2018, 121, 062501.

64. Siciliano, M.; Valiente-Dobón, J.J.; Goasduff, A.; et al. Pairing-quadrupole interplay in the neutron-deficient tin nuclei: First lifetime measurements of low-lying states in ${ }^{106,108}$ Sn. Phys. Lett. B 2020, 806, 135474.

65. Zuker, A.P. Quadrupole dominance in the light Sn and in the Cd isotopes. Phys. Rev. C 2021, 103, 024322.

66. Dufflo, J.; Zuker, A.P. The nuclear monopole Hamiltonian. Phys. Rev. C 1999, 59, R2347.

67. Fielding, H.W; Anderson, R.E.; Zafiratos, C.D.; et al. A study of the $\left({ }^{3} \mathrm{He}, n\right)$ reaction on isotopes of tin. Nucl. Phys. A 1977, 281, 389.

68. Wrzosek-Lipska, K.; Próchniak, L.; Garrett, P.E.; et al. Quadrupole deformation of ${ }^{110}$ Cd studied with Coulomb excitation. Acta Phys. Pol. B 2020, 51, 789.

69. Ekström, A.; Cederkäll, J.; DiJulio, D.D.; et al. Electric quadrupole moments of the $2_{1}^{+}$states in ${ }^{100,102,104}$ Cd. Phys. Rev. C 2009, 80 , 054302.

70. Boelaert, N.; Smirnova, N.; Heyde, K; Jolie, J. Shell model description of the low-lying states of the neutron deficient Cd isotopes. Phys. Rev. C 2007, 75, 014316.

71. Rhodes, D; Brown, B.A.; Henderson, J.; et al. Exploring the role of high-j configurations in collective observables through the Coulomb excitation of ${ }^{106}$ Cd. Phys. Rev. C 2021, 103, L051301.

72. Klintefjord, M.; Hadyńska-Klęk, K.; Görgen, A.; et al. Structure of low-lying states in ${ }^{140}$ Sm studied by Coulomb excitation. Phys. Rev. C 2016, 93, 054303.

73. Caurier, E.; Nowacki, F.; Poves, A.; Sieja, K. Collectivity in the light xenon isotopes: A shell model study. Phys. Rev. C 2010, 82, 064304.

74. Brown, B.A.; Stone, N.J.; Stone, J.R.; et al. Magnetic moments of the $2_{1}^{+}$states around ${ }^{132}$ Sn. Phys. Rev. C 2005, $71,044317$.

75. Kaia, L.; Vogt, A.; Reiter, P.; et al. Millisecond $23 / 2^{+}$isomers in the $N=79$ isotones ${ }^{133}$ Xe and ${ }^{135}$ Ba. Phys. Rev. C 2018, $98,054312$.

76. Vogt, A.; Birkenbach, B.; Reiter, P.; et al. Isomers and high-spin structures in the $N=81$ isotones ${ }^{135}$ Xe and ${ }^{137}$ Ba. Phys. Rev. C 2017, 95, 024316.

77. CUORE Collaboration: Arnaboldi, C.; Avignone III, F.T.; Beeman, J.; et al. CUORE: a cryogenic underground observatory for rare events. Nucl. Instrum. Meth. Phys. Res. A 2004, 518, 775.

78. SNO+ Collaboration: Albanese, V.; Alves, R.; Anderson, M.R.; et al. The SNO+ experiment. arXiv:2104.11687 [physics.ins-det] J. Instrum., accepted.

79. Coraggio, L.; De Angelis, L.; Fukui, T.; et al. Calculation of Gamow-Teller and two-neutrino double- $\beta$ decay properties for ${ }^{130}$ Te and ${ }^{136}$ Xe with a realistic nucleon-nucleon potential. Phys. Rev. C 2017, 95, 064324.

80. Paschalis, S.; Lee, I.Y.; Macchiavelli, A.O.; et al. The performance of the Gamma-Ray Energy Tracking In-beam Nuclear Array GRETINA. Nucl. Instrum. Methods Phys. Res. A 2013, 709, 44.

81. Surman, R.; Engel, J.; Bennett, J.R; Meyer, B.S. Source of the rare-earth element peak in r-process nucleosynthesis. Phys. Rev. Lett. $1997,79,1809$. 